\title{
Genetics and genomics of pulmonary arterial hypertension
}

\author{
Nicholas W. Morrell ${ }^{1}$, Micheala A. Aldred ${ }^{2}$, Wendy K. Chung ${ }^{3}$, \\ C. Gregory Elliott ${ }^{4}$, William C. Nichols ${ }^{5}$, Florent Soubrier $\mathbb{1}^{6}$, \\ Richard C. Trembath ${ }^{7}$ and James E. Loyd ${ }^{8}$
}

Number 2 in the series

"Proceedings of the 6th World Symposium on Pulmonary Hypertension" Edited by N. Galiè, V.V. McLaughlin, L.J. Rubin and G. Simonneau

\begin{abstract}
Affiliations: 'University of Cambridge School of Clinical Medicine, Addenbrooke's and Papworth Hospitals, Cambridge, UK. ${ }^{2}$ Indiana University School of Medicine, Indianapolis, IN, USA. ${ }^{3}$ Columbia University Medical Center, New York, NY, USA. ${ }^{~ I n t e r m o u n t a i n ~ M e d i c a l ~ C e n t e r ~ a n d ~ U n i v e r s i t y ~ o f ~ U t a h, ~ S a l t ~ L a k e ~ C i t y, ~ U T, ~ U S A ~}$ ${ }^{5}$ Cincinnati Children's Hospital Medical Center, Cincinnati, OH, USA. ${ }^{6}$ Dept of Genetics, Hopital PitiéSalpêtrière, Paris, France. ${ }^{7}$ Division of Genetics and Molecular Medicine, School of Basic and Medical Biosciences, King's College London, London, UK. ${ }^{8}$ Vanderbilt University Medical Center, Nashville, TN, USA.
\end{abstract}

Correspondence: Nicholas W. Morrell, Dept of Medicine, University of Cambridge School of Clinical Medicine, Box 157, Addenbrooke's Hospital, Hills Road, Cambridge, CB2 OQQ, UK. E-mail: nwm23acam.ac.uk

Correspondence: James E. Loyd, Division of Allergy, Pulmonary and Critical Care Medicine, Dept of Medicine, Vanderbilt University Medical Center, 1611 21st Avenue South, T-1218 Medical Center North, Nashville, TN 37232, USA. E-mail: jim.loydavanderbilt.edu

@ERSpublications

State of the art and research perspectives in genetics and genomics of pulmonary hypertension and insights into pathobiology http://ow.ly/dkkq30mgDo2

Cite this article as: Morrell NW, Aldred MA, Chung WK, et al. Genetics and genomics of pulmonary arterial hypertension. Eur Respir J 2019; 53: 1801899 [https://doi.org/10.1183/13993003.01899-2018].

ABSTRACT Since 2000 there have been major advances in our understanding of the genetic and genomics of pulmonary arterial hypertension (PAH), although there remains much to discover. Based on existing knowledge, around 25-30\% of patients diagnosed with idiopathic $\mathrm{PAH}$ have an underlying Mendelian genetic cause for their condition and should be classified as heritable PAH (HPAH). Here, we summarise the known genetic and genomic drivers of $\mathrm{PAH}$, the insights these provide into pathobiology, and the opportunities afforded for development of novel therapeutic approaches. In addition, factors determining the incomplete penetrance observed in HPAH are discussed. The currently available approaches to genetic testing and counselling, and the impact of a genetic diagnosis on clinical management of the patient with PAH, are presented. Advances in DNA sequencing technology are rapidly expanding our ability to undertake genomic studies at scale in large cohorts. In the future, such studies will provide a more complete picture of the genetic contribution to $\mathrm{PAH}$ and, potentially, a molecular classification of this disease. 


\section{Introduction}

For half a century after the mid-1900s, when cardiac catheterisation first provided clinicians the ability to safely measure pulmonary haemodynamics, idiopathic pulmonary arterial hypertension (IPAH, then termed primary pulmonary hypertension (PPH)) was recognised as a lethal pulmonary vascular disease of enigmatic origin. Near the turn of this century, new understanding was developed of the specific genetic predisposition in families with $\mathrm{PAH}$ and that information was a driver to revise the classification of PAH by the 3rd World Symposium on Pulmonary Hypertension in 2003. Since 2000, investigators have contributed increasing knowledge of the genetics and genomics of PAH. Technological advances in genetic sequencing have enabled inexpensive and rapid sequencing of the coding regions of the genome (whole exome sequencing (WES)), or the whole genome (whole genome sequencing (WGS)), in families and large cohorts of patients (figure 1). Insights from human genetics are increasing our understanding of the pathobiology of $\mathrm{PAH}$, identifying new potential drug targets, and informing the care of patients and their families.

\section{State of the art}

\section{Mendelian inheritance}

There is a family history of $\mathrm{PAH}$ in $6-10 \%$ of patients not associated with other underlying disorders [1]. In 2000, genetic analysis of such families identified heterozygous germline mutations in BMPR2, the gene encoding bone morphogenetic protein receptor type 2 , a member of the transforming growth factor- $\beta$ (TGF- $\beta$ ) superfamily [2, 3]. Subsequently, mutations were also identified in IPAH [4]. It is now well established that around $70-80 \%$ of families with PAH and $10-20 \%$ of IPAH cases are caused by mutations in BMPR2 [5].

Sequencing of genes encoding BMP receptor signalling intermediaries led to the identification of rare sequence variants in SMAD1, SMAD4 and SMAD9 [6,7]. The identification of additional SMAD9 mutations in large cohorts has confirmed its role in PAH [8]. In addition, exome sequencing of BMPR2-negative individuals with more than one family member diagnosed with PAH revealed mutations in CAV1, which encodes caveolin-1 and functions to physically colocalise BMP receptors [9]. A rare mutation in CAV1 has been associated with both lipodystrophy and PAH in a young child. KCNK3 (potassium channel subfamily $\mathrm{K}$ member 3 ) mutations were also identified by exome sequencing and encode a potassium channel that contributes to the membrane potential to determine pulmonary vascular tone [10]. Array comparative hybridisation and sequencing in childhood and (rarely) adult-onset PAH identified deletions and loss-of-function mutations in TBX4 (T-box 4), a gene that is also associated with small patella syndrome [11]. Mutations in TBX4 are among the most common genetic causes of PAH in children and suggest that PAH is at least in part a developmental lung disease when it presents early in life $[12,13]$. The recognition that severe PAH can also occur in families segregating hereditary haemorrhagic telangiectasia (HHT) implicated ACVRL1 (activin receptor-like kinase 1 (ALK1)) and ENG (endoglin) mutations in PAH [14-16].

Recently, a large survey was undertaken in a collaborative European cohort of adult-onset ( $>18$ years) patients with IPAH, familial PAH (FPAH) and anorexigen-associated PAH [8]. This study of over 1000

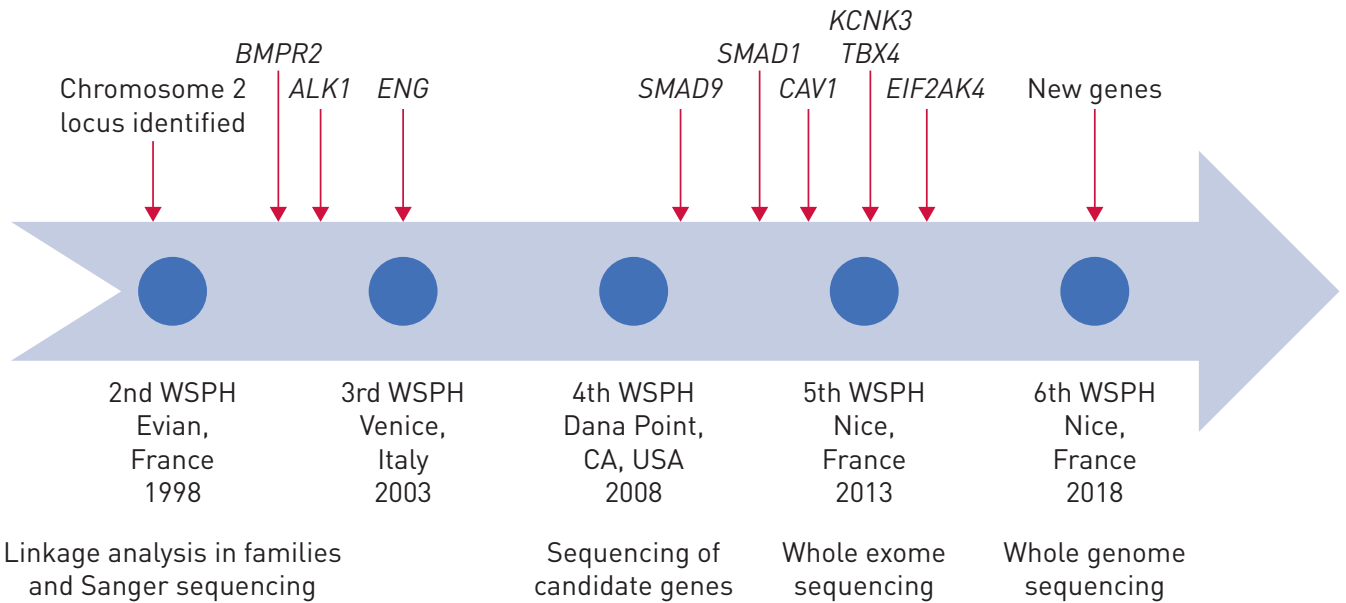

FIGURE 1 The history of genetic discovery in pulmonary arterial hypertension. WSPH: World Symposium on Pulmonary Hypertension. 
TABLE 1 Classification of pulmonary arterial hypertension genes according to level of evidence that they play a causal role in the disease

Higher level of evidence

Lower level of evidence

BMPR2; EIF2AK4; TBX4; ATP13A3; GDF2; SOX17; AQP1; ACVRL1; SMAD9; ENG; KCNK3; CAV1

SMAD4; SMAD1; KLF2; BMPR1B; KCNA5

Evidence includes de novo mutation, cosegregation studies, association with replication and functional studies.

patients confirmed the presence of causal mutations in BMPR2 (15.3\%), TBX4 (1.3\%), ACVRL1 (0.9\%), ENG (0.6\%), SMAD9 (0.4\%) and KCNK3 (0.4\%). No pathogenic coding variants in CAV1, SMAD1 or SMAD4 were identified, possibly due to the rarity of mutations in these genes in adults. The same study identified mutations in new PAH genes: ATP13A3 (ATPase 13A3; 1.1\%), SOX17 (SRY-box 17; 0.9\%), $A Q P 1$ (aquaporin $1 ; 0.9 \%$ ) and GDF2 (growth differentiation factor 2/BMP9; 0.8\%), and suggested additional genes that will require further validation. Mutations in all of these genes are autosomal dominantly inherited and exhibit reduced penetrance, meaning that some individuals who carry a mutation do not manifest PAH. In IPAH cases, the mutation may be inherited from an unaffected parent or occur de novo. Where parental samples are available, determining that a mutation occurred de novo can be helpful in establishing pathogenicity. Among paediatric IPAH patients without mutations in known risk genes, exome sequencing revealed a 2 -fold enrichment of de novo predicted deleterious variants. De novo variants in novel genes may explain $19 \%$ of paediatric-onset IPAH cases [12]. The incomplete penetrance observed for PAH genes suggests that additional genetic, epigenetic and/or environmental factors contribute to disease risk/progression.

In 2014, biallelic mutations in EIF2AK4, a gene encoding eukaryotic translation initiation factor $2 \alpha$ kinase 4 , were identified as a cause of heritable pulmonary capillary haemangiomatosis ( $\mathrm{PCH})$ [17] and pulmonary veno-occlusive disease (PVOD) [18]. PVOD and PCH are rare and pathologically distinct forms of PAH. Heritable PVOD and PCH are autosomal recessive and nearly completely penetrant, unlike other forms of $\mathrm{PAH}$. Individuals diagnosed with apparently sporadic PVOD or PCH may also carry biallelic EIF2AK4 mutations in up to $25 \%$ of cases $[17,18]$. Detection of biallelic pathogenic EIF2AK4 mutations establishes a precise and accurate molecular diagnosis of $\mathrm{PVOD} / \mathrm{PCH}$ without requiring a lung biopsy [19].

A summary of the genes reported to date in patients with HPAH is shown in table 1. A high level of evidence needs to be established for the causal role of mutations in a particular gene before it is used in clinical screening and management.

\section{Common genetic variation}

The role of common genetic variation contributing to the aetiology or clinical course of PAH is less well defined. To date only one genome-wide association study has been published to identify variants associated with PAH ( $\mathrm{n}=625$ cases). This study detected a significant association at the CBLN2 (cerebellin 2 precursor) locus mapping to $18 \mathrm{q} 22.3$, with the risk allele conferring an odds ratio for PAH of 1.97 $\left(\mathrm{p}=7.47 \times 10^{-10}\right)[20]$. Another large study evaluated associations between polymorphisms in genes comprising the endothelin signalling pathway and clinical outcomes in $715 \mathrm{PAH}$ patients of European descent in the STRIDE study (sitaxsentan). An association was identified between a single nucleotide polymorphism (rs11157866) in the G-protein $\gamma$ subunit gene GNG2 and a combined improvement in functional class and 6-min walk distance [21]. In a smaller study, levels of serum endostatin, a potent antiangiogenic protein with the capacity to induce endothelial cell apoptosis and inhibit endothelial cell proliferation, correlated with poor functional status and was a strong predictor of mortality in group 1 patients. In contrast, a missense variant (rs12483377) in COL18A1, which encodes endostatin, was associated with lower circulating protein and reduced mortality [22]. Abnormalities of mitochondrial metabolism are now well recognised in PAH. A recent study suggests that variants in the mitochondrial genome may influence the risk of developing $\mathrm{PAH}$, with a lower rate of disease associated with haplogroup $\mathrm{L}$, the oldest ancestral human haplogroup [23]. Lastly, in a study of 2761 healthy adults, variants in oestradiol metabolism (CYP1B1 (cytochrome P450 1B1)) and androgen receptor genes were associated with right ventricular function [24]. Given the significant sex disparities in $\mathrm{PAH}$ and previous evidence that CYP1B1 variants may modulate disease penetrance in BMPR2 mutation carriers [25], these variants warrant further study regarding a possible role in right ventricular remodelling in $\mathrm{PAH}$. 


\section{Pathobiology links between mutations and disease}

\section{PAH mutations and disease pathobiology}

BMPR2 is expressed on the surface of a wide number of cells, but it is particularly highly expressed on the pulmonary vascular endothelium, where it forms a complex with the type I receptors, ALK1 or ALK2. The ALK1/BMPR2 receptor complex signals specifically in response to the circulating BMP ligands, BMP9 and BMP10, utilising ENG as a coreceptor [26, 27]. ALK1 and ENG are also highly expressed in the pulmonary endothelium, and this requirement for high levels of BMPR2/ALK1 signalling in the pulmonary endothelium may contribute to the lung-specific effects of BMPR2 mutations. BMP9 (encoded by GDF2) functions as a circulating vascular quiescence factor, protects endothelial cells from apoptosis and excessive proliferation, and inhibits vascular permeability. Loss of BMPR2 also favours endothelial dysfunction and promotes endothelial-to-mesenchymal transition. Given that mutations in $A L K 1$ and ENG (usually associated with HHT) can also cause PAH, the genetic evidence strongly suggests that the pulmonary endothelial cell is an important initiating cell type in PAH pathobiology. Nevertheless, loss of BMPR2 function in other cell types (e.g. smooth muscle cells, fibroblasts and immune cells) may also contribute to disease pathobiology. For example, pulmonary artery smooth muscle cells with BMPR2 mutations are hyperproliferative and resistant to the growth-suppressive effects of BMPs, through loss of antiproliferative Smad1/5 signalling [28].

Other less common PAH mutations are also related to the BMP pathway. Smad8 (encoded by the SMAD9 gene) is a downstream mediator of BMP signalling, together with Smad1 and 5. Heterozygous mutations of SMAD9 have relatively little effect on canonical BMP signalling through Smad4, presumably due to redundancy with Smad1/5 function [29]. However, in relation to a Smad4-independent pathway that promotes microRNA maturation [30], SMAD9 mutations lead to significant loss of function, suggesting that microRNAs regulated in this manner may play an important role in PAH pathogenesis [29]. Similar results were seen in a case with congenital heart disease (CHD)-associated PAH in which pulmonary artery endothelial cells carried a somatic heterozygous deletion of SMAD9 [31].

EIF2AK4, also known as general control non-derepressible 2 (GCN2), is a serine/threonine protein kinase that phosphorylates the $\alpha$ subunit of eukaryotic initiation factor 2, which plays a key role in modulating amino acid metabolism in response to nutrient deprivation. EIF2AK4 senses amino acid deficiency through binding to uncharged transfer RNA. The molecular and cellular mechanisms by which loss of function of this kinase promotes the development of $\mathrm{PVOD} / \mathrm{PCH}$ is under active investigation. In the Eif $2 a \mathrm{k}^{-/-}$mouse, an increased inflammatory response to stress was observed in the intestine, secondary to decreased autophagy and increased oxidative stress and its effect on inflammasome activation and inflammation [32]. It remains to be demonstrated if similar mechanisms are involved in the intimal fibrosis and endothelial cell proliferation observed in the lung vessels of heritable PVOD. Alternatively, EIF2AK4 biallelic loss of function leads to decreased TRIB3 (Tribbles-like protein 3), whose downregulation has been shown to inhibit BMP-mediated cellular response [33]. Under this hypothesis, EIF2AK4 loss of function would have similar consequences to BMPR2 or SMAD9 mutations by decreasing BMP signalling.

CAV1 is a major constituent protein of caveolae (flask-shaped invaginations of the plasma membrane) and is highly expressed in endothelial cells. BMP receptors are localised in caveolae, as well as other regions of the plasma membrane, and studies suggest that caveolae are required for initiation of BMP signalling [34]. Loss of CAV1 decreases BMPR2 membrane localisation and signalling [35]. Conversely, BMPR2 mutations can lead to caveolar trafficking defects and intracellular localisation [36]. Lastly, it is notable that even in the absence of a heritable mutation, expression levels of BMPR2 and CAV1 are reduced in PAH lung tissues $[37,38]$.

\section{Factors affecting disease penetrance}

Despite the fact that pathogenic BMPR2 mutations clearly cause PAH, the penetrance of the disease phenotype is incomplete. The best estimate is that penetrance in male carriers is around $14 \%$, whereas in females it is around $42 \%$ [39]. Thus, female sex is the single most important factor influencing the penetrance of BMPR2 mutations in $\mathrm{PAH}$, possibly driven by oestrogen metabolism [25]. Additional factors influencing penetrance may be genetic, epigenetic and/or environmental. Genetic factors may include the expression level of wild-type $B M P R 2$ from the unaffected allele [40], genetic variants affecting the expression levels of TGF- $\beta$ [41] or alternative splicing of BMPR2 [42]. Studies in induced pluripotent stem cells derived from affected and unaffected BMPR2 mutation carriers have suggested that genetic background, specifically differences in expression of BMP pathway-modifying genes, might contribute to penetrance [43]. Additional large-scale longitudinal studies of affected and unaffected mutation carriers are required to more systematically search for genetic and environmental modifiers of penetrance. 
Although no study has yet addressed potential environmental factors influencing the penetrance of BMPR2 mutations in human cohorts, studies suggest that inflammatory mediators, e.g. lipopolysaccharide [44], tumour necrosis factor- $\alpha$ (TNF- $\alpha)$ [45] and 5-lipoxygenase [46], can drive the development of $\mathrm{PH}$ in genetically modified mice and in patient-derived pluripotent stem cells [47]. TNF- $\alpha$ directly suppresses $B M P R 2$ mRNA and protein expression. In addition, epigenetic mechanisms such as hypermethylation of the $B M P R 2$ promoter may play a role [48]. Several microRNAs have also been shown to target BMPR2 mRNA to modify expression levels of the protein, including miR-21 [49] and miR-17-92 [50].

Another factor that may contribute to penetrance is somatic mutation within lung vascular cells [51]. Several groups have identified DNA damage in endothelial and smooth muscle cells from PAH lungs in comparison with controls [51-53]. Loss of BMPR2 was associated with a deficiency of DNA repair [54], but increased DNA damage was also evident in cells from IPAH and associated PAH cases, and may be induced by environmental exposures such as methamphetamine use [55]. Further studies are needed to understand the role of DNA damage within the lung and its contribution to disease pathogenesis.

\section{Genetic counselling/testing and management of healthy mutation carriers Genetic counselling}

Mutations in PAH genes have been identified in IPAH and FPAH, anorexigen-associated PAH, PVOD/ $\mathrm{PCH}$, and in children with IPAH and PAH associated with $\mathrm{CHD}$. There is a medicolegal duty to inform all patients in these groups about the possibility of a genetic condition and that family members could carry a mutation that increases the risk of $\mathrm{PAH}$, allowing for screening and early diagnosis. Even if genetic testing is not performed, family members should be made aware of early signs and symptoms to ensure that a timely and appropriate diagnosis is made. If a mutation is identified in a patient, the symptomatic patient should be reclassified as HPAH.

Genetic education and counselling should be performed prior to genetic testing for PAH to address the complex issues of incomplete penetrance, questions of surveillance for genetically at-risk family members, reproductive questions, concerns about genetic discrimination, as well as psychosocial issues of guilt and blame that can accompany genetically based diseases. Pre-test genetic education of the affected individual can be performed by PAH providers and/or genetic professionals, and is facilitated by focused educational videos (e.g. www.youtube.com/watch? $\mathrm{v}=36 \mathrm{rlvtj}$ Q Qrs). In-depth genetic counselling with genetic professionals including genetic counsellors or medical geneticists is critical prior to genetic testing for asymptomatic family members. Families should also be referred to a genetic counsellor and/or clinical geneticist if they wish to consider reproductive options. The genetic counselling experience of a national reference centre on $\mathrm{PAH}$ shows the potential interest in genetic testing in asymptomatic family members [56].

Genetic testing in the family should begin with an affected individual whenever possible to identify the relevant mutation in the family. Otherwise, a negative genetic test result in unaffected family members is not informative. If the familial mutation is known and an unaffected family member tests negative for that mutation, the risk of PAH for that person is the same as the general population (around 1 per 1000000 in North America). This can provide great psychological relief to that family member and they can forgo evaluations to screen for PAH.

\section{Genetic testing}

Genetic testing can help to explain the aetiology of the disease and stratify risk for other family members and for future children. Clinical genetic testing is available in North America and Europe, and material can be sent to American or European genetic laboratories from other parts of the world. The current cost of testing ranges from approximately USD1000 to USD1500 to analyse the first member of a family. Once the family-specific mutation is known, testing other family members costs USD300-500 in the USA. The exact number of genes included, cost of testing and insurance coverage for testing varies by country and insurer. The most commonly cited reason for genetic testing for $\mathrm{PAH}$ is to provide information to and about children, and interest in testing is especially high for paediatric-onset PAH [57].

\section{Targeted sequencing}

As the number of genes associated with PAH increases, it has become arduous to test for each of these genes individually. The advent of next-generation sequencing has enabled the development of gene panels to interrogate several genes simultaneously. Several different technologies and platforms are available, and many are now offered on a clinical basis (www.ncbi.nlm.nih.gov/gtr/conditions/C0152171/). In addition, several of these genes can be analysed for dosage changes to detect deletions or duplications of one or more exons. Such mutations are common in BMPR2 and TBX4. An appropriate methodology, such as multiplex ligation-dependent probe amplification, should be used since these types of mutations are not 
readily detected by sequencing. It is important to check the genes included in the panel at the time of testing since the composition changes as genetic discoveries advance.

Whole exome sequencing and whole genome sequencing

If an up-to-date panel of PAH genes fails to identify a pathogenic variant in a patient with familial PAH or in a child, WES may be appropriate to identify potentially novel genes. In the case of paediatric-onset $\mathrm{PAH}$, testing should ideally include the child and both parents to allow for facile identification of de novo variants. This trio approach was used to identify both the CAV1 and $K C N K 3$ genes $[9,10]$. The largest trio analyses suggest that $19 \%$ of childhood-onset IPAH is due to de novo mutations [12]. Exome sequencing is significantly more expensive than panel gene testing and should be ordered by a genetic professional who can discuss the option of learning about incidental or secondary findings, currently comprised of 59 genes recommended by the American College of Medical Genetics and Genomics that are medically actionable and potentially life saving, including genes for hereditary cancer and sudden cardiac death [58].

On the horizon is the use of WGS, which enables the identification of variants in the non-coding regions in a patient's genome, but is not yet routinely used on a clinical basis. Exome and genome sequencing have the advantage of iterative reanalysis over time as novel genes are identified.

\section{Interpretation of sequencing data}

The main problem with WES and WGS is the large number of variants identified in any individual. Interpreting the functional consequences of missense and non-coding variants is particularly challenging. Therefore, identifying potentially pathogenic variants in novel genes requires filtering out common variants based upon large databases of reference allele frequencies and bioinformatic prediction of the likely functional effects. Segregation studies within families can sometimes clarify a variant of uncertain significance (VUS), especially if the variant is de novo or if it segregates with PAH in other affected family members [58], while appreciating that penetrance is usually incomplete and that carriers may be unaffected. Only genetic variants classified as pathogenic or likely pathogenic (not VUS) can be used to genetically risk stratify unaffected family members.

\section{Psychosocial considerations and reproductive options}

Genetic test results may cause more harm than good in some individuals because there is currently no effective way to prevent or differentially treat hereditary forms of PAH and because of the incomplete penetrance of mutations. Identifying a mutation in a family can be associated with feelings of guilt in the parent who has passed on a mutation to their child. There are also concerns about genetic discrimination in employment and insurance. Legal safeguards against genetic discrimination vary by country. In the USA, the Genetic Information Nondiscrimination Act protects insured members against discrimination in coverage or cost of health insurance and protects against discrimination in employment, but not against discrimination in life, long-term care or disability insurance based upon a genetic predisposition. In countries with universal healthcare, the concerns about genetic discrimination are not as great and genetic testing uptake rates have been higher.

Reproductive options for those who carry pathogenic mutations include adoption, use of donor gametes, pre-natal testing, in vitro fertilisation (IVF) with pre-implantation genetic diagnosis or not considering PAH genetic status. Accessibility and insurance coverage for IVF and pre-implantation genetic diagnosis vary by country, but coverage is often not readily available due to the cost to the patient.

\section{Practical application of genetics and genomics}

What is the utility of genetic diagnosis in PAH patients?

Pathogenic PAH mutations associate with disease characteristics that influence treatment and prognosis. For example, HPAH associated with BMPR2 or ACVRL1 mutations presents at a younger age, with more severe haemodynamic abnormalities, and a very low probability of acute vasoreactivity, as well as reduced survival in the current treatment era $[59,60]$. Patients with a heritable form of PVOD are also younger at presentation than non-mutation carriers, but there is no significant difference in the event-free survival at 3 years [61].

Several studies have clearly shown that detection of pathogenic EIF2AK4 mutations is especially useful. The diagnosis of $\mathrm{PVOD} / \mathrm{PCH}$ challenges $\mathrm{PH}$ clinicians, radiologists and pathologists. Identification of biallelic EIF2AK4 mutations allows confirmation of heritable PVOD/PCH without a lung biopsy and identifies a unique form of PAH that not only does not respond well to current PAH medication, but also predisposes to pulmonary oedema when these medications are administered [61]. A subgroup of individuals diagnosed with IPAH or HPAH may also carry biallelic pathogenic EIF2AK4 mutations. These individuals are characterised by presenting under the age of 50 years with a diffusing capacity of the lung 
for carbon monoxide (DLCO) $<50 \%$ of predicted [62-64]. Thus, we suggest that clinicians test younger PAH patients who have a low DLCO for EIF2AK4 mutations in order to guide treatment and for risk assessment in family members [63].

Findings from genomic studies are also starting to be used to better understand the disease process and therapeutic responses. Hemnes et al. [65] identified a peripheral blood RNA expression signature in patients with a $\mathrm{PAH}$ subphenotype responsive to calcium channel blocker therapy, suggesting a non-invasive approach to predict drug responsiveness. Using WES, genetic variants that may underlie this subphenotype were also identified [66]. As discussed previously, common genetic variants have been associated with risk of $\mathrm{PAH}$, survival and/or pharmacogenomic response to therapy. Most recently, it was shown that common functional variants in SIRT3 and UCP2 might predict the response to inhibition of pyruvate dehydrogenase kinase by dichloroacetate in a phase 2 clinical trial in $\mathrm{PAH}$ [67], paving the way for future precision medicine studies with this drug.

\section{From genes to therapies}

Molecular pathways highlighted by genetic studies are now the subject of several novel therapeutic approaches (figure 2). LoNG et al. [68] reported the beneficial effects of BMP9 administration in heterozygous BMPR2 knockout mice, suggesting that compensating for BMPR2 haploinsufficiency by increasing dose of the ligand might constitute a targeted therapy for human PAH. At the receptor level, approaches include translational readthrough of nonsense mutations using ataluren to restore full-length protein [69] or slowing down lysosomal degradation of BMPR2 with chloroquine to increase receptor density at the cell surface $[70,71]$. Both approaches effectively restored BMP signalling in vitro and lysosomal blockade reversed $\mathrm{PH}$ in experimental models. Inhibition of TNF- $\alpha$ with etanercept not only

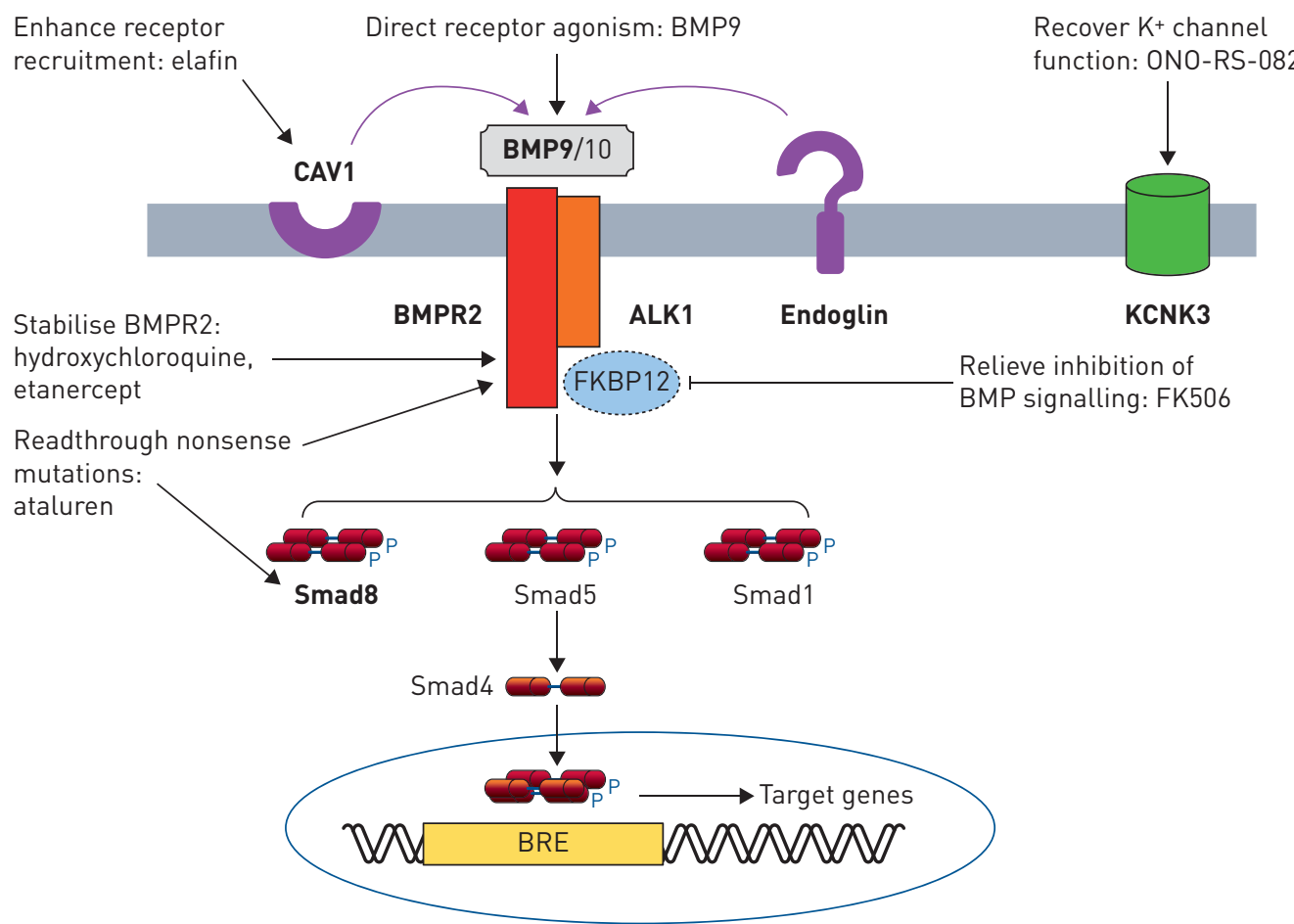

FIGURE 2 From genes to therapies: precision medicine approaches in pulmonary arterial hypertension (PAH). BMP(R): bone morphogenetic protein (receptor); BRE: BMP-responsive element; CAV1: caveolin-1; FKBP12: $12-k D a$ FK506-binding protein. BMP signalling in endothelial cells is mediated by the ligands BMP9 and BMP10 via the ALK1/BMPR2 receptor complex. Endoglin serves as an accessory receptor. Signalling is mediated by phosphorylation of the receptor Smads (Smad1, 5 and 8), which then associate with Smad4 and translocate to the nucleus, regulating genes that contain BREs in their promoters. CAV1 facilitates receptor colocalisation, while KCNK3 encodes a potassium channel that contributes to pulmonary vascular tone. Genes that are mutated in heritable PAH are in bold. Potential therapeutic approaches targeted to these pathways include: exogenous administration of BMP9 ligand, increasing availability of functional BMPR2 receptors (hydroxychloroquine, etanercept), promoting readthrough of nonsense mutations to restore functional BMPR2 or Smad8 protein lataluren), enhancing downstream signalling by relieving FKBP12 inhibition of BMP type 1 receptors (FK506), promoting CAV1-mediated receptor recruitment (elafin), or recovering KCNK3 channel conductance (ONO-RS-082). 
targets inflammation, but can reduce receptor shedding and proteosomal degradation of BMPR2 [45]. Elafin acts via CAV1 to promote receptor recruitment and enhance BMP signalling, reversing experimental $\mathrm{PH}$ [72]. Downstream of BMPR2, FK506 was found to increase BMP signalling and to reverse experimental $\mathrm{PH}$, by binding FK-binding protein 12, an inhibitor of BMP signalling [73]. Phase 2a clinical trials have established safety and tolerability of low-dose FK506 in PAH [74]. Beyond the BMP pathway, KCNK3 mutations lead to reduced potassium channel conductance, which, at least for some mutations, can be recovered by the phospholipase $A_{2}$ inhibitor ONO-RS-082 [10]. These studies underline the considerable progress that has been made in translating basic genetic studies into potential therapeutic modalities. While direct correction of the underlying mutations remains challenging at present, rapid advances in gene-editing technologies may make targeted mutational correction within the lung vasculature a realistic prospect in the future.

\section{Proposed future directions}

These different genomic approaches highlight potential paths forward for using molecular medicine to improve PAH patient care. Several large-scale genetics/genomics studies are currently underway in the USA and Europe. Of note, the US National Biological Sample and Data Repository for PAH (the "PAH Biobank"; www.pahbiobank.org) is generating genetic data (targeted DNA sequencing, WES and genome-wide single nucleotide polymorphisms) for 3000 group 1 PAH patients. The BRIDGE Project in the UK includes PAH as one of the rare diseases for which WGS data are being generated (https:// bridgestudy.medschl.cam.ac.uk/pah.shtml). Over $1250 \mathrm{IPAH} /$ familial PAH patients from Europe are being analysed to investigate the underlying genetic variation contributing to the disease. In addition, the US National Institutes of Health PVDOMICS initiative aims to define new molecular classifications across the traditional World Health Organization groups by combining deep clinical phenotyping with multiple "omics" analyses [75]. It is only through such cohorts and initiatives that we will begin to have adequate power to discover the range of genetic factors underlying $\mathrm{PAH}$ to provide a more complete genomic understanding of the disease. Ideally, this will lead to the identification of additional novel targets for new drugs. It also may facilitate prevention strategies for $\mathrm{PAH}$ and the prediction of prognosis based on a genetic classification of PAH.

Conflict of interest: N.W. Morrell reports grants and personal fees from Morphogen-IX, outside the submitted work. M.A. Aldred reports grants from the NIH, during the conduct of the study. W.K. Chung has nothing to disclose. C.G. Elliott reports personal fees for steering committee work from Bayer and Bellerophon, grants and personal fees for registry and data safety monitoring from Actelion, and was a consultant for end-point adjudication for Lung LLC, with fees paid to his employer (Intermountain Healthcare), outside the submitted work. W.C. Nichols has nothing to disclose. F. Soubrier has nothing to disclose. R.C. Trembath has nothing to disclose. J.E. Loyd has nothing to disclose.

\section{References}

1 Loyd JE, Primm RK, Newman JH. Familial primary pulmonary hypertension: clinical patterns. Am Rev Respir Dis 1984; 129: 194-197.

2 Lane KB, Machado RD, Pauciulo MW, et al. Heterozygous germline mutations in BMPR2. Nat Genet 2000; 26: 81-84.

3 Deng Z, Morse JH, Slager SL, et al. Familial primary pulmonary hypertension (gene PPH1) is caused by mutations in the bone morphogenetic protein receptor-II gene. Am J Hum Genet 2000; 67: 737-744.

4 Thomson JR, Machado RD, Pauciulo MW, et al. Sporadic primary pulmonary hypertension is associated with germline mutations of the gene encoding BMPR-II. J Med Genet 2000; 37: 741-745.

5 Evans JD, Girerd B, Montani D, et al. BMPR2 mutations and survival in pulmonary arterial hypertension: an individual participant data meta-analysis. Lancet Respir Med 2016; 4: 129-137.

6 Nasim MT, Ogo T, Ahmed M, et al. Molecular genetic characterization of SMAD signaling molecules in pulmonary arterial hypertension. Hum Mutat 2011; 32: 1385-1389.

7 Shintani M, Yagi H, Nakayama T, et al. A new nonsense mutation of SMAD8 associated with pulmonary arterial hypertension. J Med Genet 2009; 46: 331-337.

8 Gräf S, Haimel M, Bleda $\mathrm{M}$, et al. Identification of rare sequence variation underlying heritable pulmonary arterial hypertension. Nat Commun 2018; 9: 1416.

9 Austin ED, Ma L, LeDuc C, et al. Whole exome sequencing to identify a novel gene (caveolin-1) associated with human pulmonary arterial hypertension. Circ Cardiovasc Genet 2012; 5: 336-343.

$10 \mathrm{Ma}$ L, Roman-Campos D, Austin ED, et al. A novel channelopathy in pulmonary arterial hypertension. $N$ Engl $J$ Med 2013; 369: 351-361.

11 Kerstjens-Frederikse WS, Bongers EM, Roofthooft MT, et al. TBX4 mutations (small patella syndrome) are associated with childhood-onset pulmonary arterial hypertension. J Med Genet 2013; 50: 500-506.

12 Zhu N, Gonzaga-Jauregui C, Welch CL, et al. Exome sequencing in children with pulmonary arterial hypertension demonstrates differences compared with adults. Circ Genom Precis Med 2018; 11: e001887.

13 Levy M, Eyries M, Szezepanski I, et al. Genetic analyses in a cohort of children with pulmonary hypertension. Eur Respir J 2016; 48: 1118-1126.

14 Trembath RC, Thomson JR, Machado RD, et al. Clinical and molecular genetic features of pulmonary hypertension in patients with hereditary hemorrhagic telangiectasia. N Engl J Med 2001; 345: 325-334. 
15 Harrison RE, Berger R, Haworth SG, et al. Transforming growth factor-beta receptor mutations and pulmonary arterial hypertension in childhood. Circulation 2005; 111: 435-441.

16 Chaouat A, Coulet F, Favre C, et al. Endoglin germline mutation in a patient with hereditary haemorrhagic telangiectasia and dexfenfluramine associated pulmonary arterial hypertension. Thorax 2004; 59: 446-448.

17 Best DH, Sumner KL, Austin ED, et al. EIF2AK4 mutations in pulmonary capillary hemangiomatosis. Chest 2014; 145: 231-236.

18 Eyries M, Montani D, Girerd B, et al. EIF2AK4 mutations cause pulmonary veno-occlusive disease. Nat Genet 2014; 46: 65-69.

19 Galiè N, Humbert M, Vachiery JL, et al. 2015 ESC/ERS Guidelines for the diagnosis and treatment of pulmonary hypertension. Eur Respir J 2015; 46: 903-975.

20 Germain M, Eyries M, Montani D, et al. Genome-wide association analysis identifies a susceptibility locus for pulmonary arterial hypertension. Nat Genet 2013; 45: 518-521.

21 Benza RL, Gomberg-Maitland M, Demarco T, et al. Endothelin-1 pathway polymorphisms and outcomes in pulmonary arterial hypertension. Am J Respir Crit Care Med 2015; 192: 1345-1354.

22 Damico R, Kolb TM, Valera L, et al. Serum endostatin is a genetically determined predictor of survival in pulmonary arterial hypertension. Am J Respir Crit Care Med 2015; 191: 208-218.

23 Farha S, Hu B, Comhair S, et al. Mitochondrial haplogroups and risk of pulmonary arterial hypertension. PLoS One 2016; 11: e0156042.

24 Ventetuolo CE, Mitra N, Wan F, et al. Oestradiol metabolism and androgen receptor genotypes are associated with right ventricular function. Eur Respir J 2016; 47: 553-563.

25 Austin ED, Cogan JD, West JD, et al. Alterations in oestrogen metabolism: implications for higher penetrance of familial pulmonary arterial hypertension in females. Eur Respir J 2009; 34: 1093-1099.

26 David L, Mallet C, Mazerbourg S, et al. Identification of BMP9 and BMP10 as functional activators of the orphan activin receptor-like kinase 1 (ALK1) in endothelial cells. Blood 2007; 109: 1953-1961.

27 Upton PD, Davies RJ, Trembath RC, et al. Bone morphogenetic protein (BMP) and activin type II receptors balance BMP9 signals mediated by activin receptor-like kinase-1 in human pulmonary artery endothelial cells. J Biol Chem 2009; 284: 15794-15804.

28 Yang X, Long L, Southwood M, et al. Dysfunctional Smad signaling contributes to abnormal smooth muscle cell proliferation in familial pulmonary arterial hypertension. Circ Res 2005; 96: 1053-1063.

29 Drake KM, Zygmunt D, Mavrakis L, et al. Altered microRNA processing in heritable pulmonary arterial hypertension: an important role for Smad-8. Am J Respir Crit Care Med 2011; 184: 1400-1408.

30 Davis BN, Hilyard AC, Lagna G, et al. SMAD proteins control DROSHA-mediated microRNA maturation. Nature 2008; 454: 56-61.

31 Drake KM, Comhair SA, Erzurum SC, et al. Endothelial chromosome 13 deletion in congenital heart disease-associated pulmonary arterial hypertension dysregulates SMAD9 signaling. Am J Respir Crit Care Med 2015; 191: 850-854.

32 Ravindran R, Loebbermann J, Nakaya HI, et al. The amino acid sensor GCN2 controls gut inflammation by inhibiting inflammasome activation. Nature 2016; 531: 523-527.

33 Chan MC, Nguyen PH, Davis BN, et al. A novel regulatory mechanism of the bone morphogenetic protein (BMP) signaling pathway involving the carboxyl-terminal tail domain of BMP type II receptor. Mol Cell Biol 2007; 27: 5776-5789.

34 Bonor J, Adams EL, Bragdon B, et al. Initiation of BMP2 signaling in domains on the plasma membrane. J Cell Physiol 2012; 227: 2880-2888.

35 Wertz JW, Bauer PM. Caveolin-1 regulates BMPRII localization and signaling in vascular smooth muscle cells. Biochem Biophys Res Commun 2008; 375: 557-561.

36 Prewitt AR, Ghose S, Frump AL, et al. Heterozygous null bone morphogenetic protein receptor type 2 mutations promote SRC kinase-dependent caveolar trafficking defects and endothelial dysfunction in pulmonary arterial hypertension. J Biol Chem 2015; 290: 960-971.

37 Atkinson C, Stewart S, Upton PD, et al. Primary pulmonary hypertension is associated with reduced pulmonary vascular expression of type II bone morphogenetic protein receptor. Circulation 2002; 105: 1672-1678.

38 Achcar RO, Demura Y, Rai PR, et al. Loss of caveolin and heme oxygenase expression in severe pulmonary hypertension. Chest 2006; 129: 696-705.

39 Larkin EK, Newman JH, Austin ED, et al. Longitudinal analysis casts doubt on the presence of genetic anticipation in heritable pulmonary arterial hypertension. Am J Respir Crit Care Med 2012; 186: 892-896.

40 Hamid R, Cogan JD, Hedges LK, et al. Penetrance of pulmonary arterial hypertension is modulated by the expression of normal BMPR2 allele. Hum Mutat 2009; 30: 649-654.

41 Phillips JA III, Poling JS, Phillips CA, et al. Synergistic heterozygosity for TGFbeta1 SNPs and BMPR2 mutations modulates the age at diagnosis and penetrance of familial pulmonary arterial hypertension. Genet Med 2008; 10: 359-365.

42 Cogan J, Austin E, Hedges L, et al. Role of BMPR2 alternative splicing in heritable pulmonary arterial hypertension penetrance. Circulation 2012; 126: 1907-1916.

43 Gu M, Shao NY, Sa S, et al. Patient-specific iPSC-derived endothelial cells uncover pathways that protect against pulmonary hypertension in BMPR2 mutation carriers. Cell Stem Cell 2017; 20: 490-504.

44 Soon E, Crosby A, Southwood M, et al. Bone morphogenetic protein receptor type II deficiency and increased inflammatory cytokine production. A gateway to pulmonary arterial hypertension. Am J Respir Crit Care Med 2015; 192: 859-872.

45 Hurst LA, Dunmore BJ, Long L, et al. TNFalpha drives pulmonary arterial hypertension by suppressing the BMP type-II receptor and altering NOTCH signalling. Nat Commun 2017; 8: 14079.

46 Song $\mathrm{Y}$, Jones JE, Beppu $\mathrm{H}$, et al. Increased susceptibility to pulmonary hypertension in heterozygous BMPR2-mutant mice. Circulation 2005; 112: 553-562.

47 Kiskin FN, Chang CH, Huang CJZ, et al. Contributions of BMPR2 mutations and extrinsic factors to cellular phenotypes of pulmonary arterial hypertension revealed by iPSC modeling. Am J Respir Crit Care Med 2018; 198: $271-275$ 
48 Liu D, Yan Y, Chen JW, et al. Hypermethylation of BMPR2 promoter occurs in patients with heritable pulmonary arterial hypertension and inhibits BMPR2 expression. Am J Respir Crit Care Med 2017; 196: 925-928.

49 Qin W, Zhao B, Shi Y, et al. BMPRII is a direct target of miR-21. Acta Biochim Biophys Sin 2009; 41: 618-623.

50 Brock M, Trenkmann M, Gay RE, et al. Interleukin-6 modulates the expression of the bone morphogenic protein receptor type II through a novel STAT3-microRNA cluster 17/92 pathway. Circ Res 2009; 104: 1184-1191.

51 Federici C, Drake KM, Rigelsky CM, et al. Increased mutagen sensitivity and DNA damage in pulmonary arterial hypertension. Am J Respir Crit Care Med 2015; 192: 219-228.

52 Meloche J, Pflieger A, Vaillancourt M, et al. Role for DNA damage signaling in pulmonary arterial hypertension. Circulation 2014; 129: 786-797.

53 de Jesus Perez VA, Yuan K, Lyuksyutova MA, et al. Whole-exome sequencing reveals TopBP1 as a novel gene in idiopathic pulmonary arterial hypertension. Am J Respir Crit Care Med 2014; 189: 1260-1272.

54 Li M, Vattulainen S, Aho J, et al. Loss of bone morphogenetic protein receptor 2 is associated with abnormal DNA repair in pulmonary arterial hypertension. Am J Respir Cell Mol Biol 2014; 50: 1118-1128.

55 Chen PI, Cao A, Miyagawa K, et al. Amphetamines promote mitochondrial dysfunction and DNA damage in pulmonary hypertension. JCI Insight 2017; 2: e90427.

56 Girerd B, Montani D, Jais X, et al. Genetic counselling in a national referral centre for pulmonary hypertension. Eur Respir J 2016; 47: 541-552.

57 Jones DL, Sandberg JC, Rosenthal MJ, et al. What patients and their relatives think about testing for BMPR2. J Genet Couns 2008; 17: 452-458.

58 Richards S, Aziz N, Bale S, et al. Standards and guidelines for the interpretation of sequence variants: a joint consensus recommendation of the American College of Medical Genetics and Genomics and the Association for Molecular Pathology. Genet Med 2015; 17: 405-424.

59 Sztrymf B, Coulet F, Girerd B, et al. Clinical outcomes of pulmonary arterial hypertension in carriers of BMPR2 mutation. Am J Respir Crit Care Med 2008; 177: 1377-1383.

60 Girerd B, Montani D, Coulet F, et al. Clinical outcomes of pulmonary arterial hypertension in patients carrying an ACVRL1 (ALK1) mutation. Am J Respir Crit Care Med 2010; 181: 851-861.

61 Montani D, Girerd B, Jais X, et al. Clinical phenotypes and outcomes of heritable and sporadic pulmonary veno-occlusive disease: a population-based study. Lancet Respir Med 2017; 5: 125-134.

62 Shi R, Ling $\mathrm{X}$, Li X, et al. Tuning hexagonal $\mathrm{NaYbF}_{4}$ nanocrystals down to sub-10 nm for enhanced photon upconversion. Nanoscale 2017; 9: 13739-13746.

63 Hadinnapola C, Bleda M, Haimel M, et al. Phenotypic characterization of EIF2AK4 mutation carriers in a large cohort of patients diagnosed clinically with pulmonary arterial hypertension. Circulation 2017; 136: 2022-2033.

64 Best DH, Sumner KL, Smith BP, et al. EIF2AK4 mutations in patients diagnosed with pulmonary arterial hypertension. Chest 2017; 151: 821-828.

65 Hemnes AR, Trammell AW, Archer SL, et al. Peripheral blood signature of vasodilator-responsive pulmonary arterial hypertension. Circulation 2015; 131: 401-409.

66 Hemnes AR, Zhao M, West J, et al. Critical genomic networks and vasoreactive variants in idiopathic pulmonary arterial hypertension. Am J Respir Crit Care Med 2016; 194: 464-475.

67 Michelakis ED, Gurtu V, Webster L, et al. Inhibition of pyruvate dehydrogenase kinase improves pulmonary arterial hypertension in genetically susceptible patients. Sci Transl Med 2017; 9: eaao4583.

68 Long L, Ormiston ML, Yang X, et al. Selective enhancement of endothelial BMPR-II with BMP9 reverses pulmonary arterial hypertension. Nat Med 2015; 21: 777-785.

69 Drake KM, Dunmore BJ, McNelly LN, et al. Correction of nonsense BMPR2 and SMAD9 mutations by ataluren in pulmonary arterial hypertension. Am J Respir Cell Mol Biol 2013; 49: 403-409.

70 Dunmore BJ, Drake KM, Upton PD, et al. The lysosomal inhibitor. Hum Mol Genet 2013; 22: 3667-3679.

71 Long L, Yang X, Southwood M, et al. Chloroquine prevents progression of experimental pulmonary hypertension via inhibition of autophagy and lysosomal bone morphogenetic protein type II receptor degradation. Circ Res 2013; 112: 1159-1170.

72 Nickel NP, Spiekerkoetter E, Gu M, et al. Elafin reverses pulmonary hypertension via caveolin-1-dependent bone morphogenetic protein signaling. Am J Respir Crit Care Med 2015; 191: 1273-1286.

73 Spiekerkoetter E, Tian X, Cai J, et al. FK506 activates BMPR2. J Clin Invest 2013; 123: 3600-3613.

74 Spiekerkoetter E, Sung YK, Sudheendra D, et al. Randomised placebo-controlled safety and tolerability trial of FK506 (tacrolimus) for pulmonary arterial hypertension. Eur Respir J 2017; 50: 1602449.

75 Hemnes AR, Beck GJ, Newman JH, et al. PVDOMICS: a multi-center study to improve understanding of pulmonary vascular disease through phenomics. Circ Res 2017; 121: 1136-1139. 